\title{
Freeze-dried Black Raspberry Bioadhesive Gel
}

National Cancer Institute

\section{Source}

National Cancer Institute. Freeze-dried Black Raspberry Bioadhesive Gel. NCI Thesaurus. Code $C 95770$.

\begin{abstract}
A bioadhesive gel containing 10\% freeze-dried black raspberries (FBR) with potential chemopreventive and antioxidant activities. The four main constituent black raspberry anthocyanins that contribute significantly to the chemopreventive effects are cyanidin 3glucoside (C3GLU), cyanidin 3-rutinoside (C3RUT), cyanidin 3-sambubioside (C3SAM) and cyanidin 3-(2(G)-xylosyl) rutinoside (C3XRUT). Upon mucosal application, the anthocyanins from the gel penetrate the oral mucosa and are able to modulate expression of certain proapoptotic and terminal differentiation genes, and reduce the expression of epithelial cyclooxygenase-2 (COX-2) protein. In addition, this gel may also reduce vascular densities in the superficial connective tissues.
\end{abstract}

